\title{
SPECTACLE OU EXPÉRIMENTATION : LA PRÉFABRICATION DE VAISSEAUX EN 1679
}

L'Ordonnance de 1689 sur la Marine est le fruit d'un travail d'enquétes et d'épreuves mené par Colbert, à partir des années 1670 et poursuivi par Seignelay. Pendant cette période, Colbert se préoccupe autant de savoir ce qu'il peut obtenir, que d'obtenir ce qu'il veut. On peut comprendre ses dispositions et décisions comme celles d'une stratégie qui vise en même temps ces deux fins. Ce n'était pas une mince gageure ; c'est pourtant celle de toute politique d'innovation.

Pour recueillir les informations utiles à ce double projet, Colbert met en place différents modes d'évaluation. Ce qui caractérise peut-être le mieux son action est le recours systématique a la confrontation d'opinions diversifices par l'origine : lieu, statut, corps... de ceux qui les émettent. Ce ne fut pas le cas, par exemple, dans la Fortification où, très tôt, l'on table sur l'expertise technique. Le partage des responsabilités et de l'autorité n'obeit pas aux mêmes clivages. Les effets de ces choix sur l'histoire des techniques constructives meriteraient sans doute un examen approfondi.

Dans la Marine, il convient d'abord de se faire une idé de l'organisation possible, puis de définir des objectifs qui y correspondent. lls sont mis autoritairement a l'épreuve et les résultats de ces essais - mais également le déroulement de leur mise en ocuvre - évalués. En suite de quoi on peut estimer la convenance des objectifs choisis par rapport à l'idee qu'on se formait de l'organisation, et donc la pertinence de cette dernière. L'extrême abstraction de ce schéma qui fonctionnera pendant tout le XVIII siécle, signale l'importance des compromis nécessaires pour sa mise en pratique. On retiendra deux difficultés : la première est que l'on exige l'avis des agents de la Marine sur l'organisation possible,

Revue de synthese : IV' S. $\mathbf{N}^{\bullet} 2$, avril-juin 1987. 
et qu'on leur impose les objectifs à remplir, une fois ceux-ci élaborés dans les bureaux du Ministère. Il faut donc obtenir conjointement des mêmes hommes, ces deux manières de contribuer à l'autorité des décisions : avoir part à leur élaboration et s'y soumettre absolument. Il est entendu qu'elles " ne doivent rendre aucune raison de leurs dispositions " ' . Encore faut-il rendre ces objectifs réalisables : ajuster les moyens aux fins, et les fins aux moyens : le "dessein " aux "faits ". Colbert procède par itération, mettant différentes solutions à l'épreuve, à l'occasion d'un projet, quitte à le rectifier en cours de route. Aux agents de contribuer encore à ces essais, en sachant acquiescer à la fois « à l'intention et aux services de Sa Majesté », comme Colbert ne manque de le rappeler.

À la lecture de sa correspondance avec les arsenaux, on mesure les difficultés de cette démarche " tâtonneuse ». Mais également, on ne peut qu'être impressionné par l'ampleur de la pensée qui la guide. Elle acquiert progressivement les traits d'une conception fonctionnelle de l'organisation. Ce qui s'exprime avec la plus grande clarté, dans les cas où Colbert s'applique à établir des règlements. On pouvait s'y attendre.

Sur la foi des philosophes, on considère l'imitation de la nature dans la production d'artifices comme la source de l'idée de fonction. Les écrits de Colbert nous invitent à penser que, dans certains cas, le modèle a transité par celui des " corps » dont on connaît l'importance sous l'Ancien Régime. Dans la Marine, on appelle d'abord «fonctions " les devoirs d'un agent. Définir les fonctions d'un Intendant, d'un Commissaire qu'il nomme à tel poste, c'est à quoi Colbert s'applique assidûment dans ses Instructions ${ }^{2}$. Il semble que ce soit pendant l'année 1670 que, par analogie, il étend l'emprise du concept : l'arsenal lui-même est conçu comme un ensemble de fonctions : magasinage, conservation des matières, constructions, fabriques... ${ }^{3}$. Dans un autre Mémoire, il systématise en définissant " les deux fonctions de la guerre de mer " ${ }^{4}$ et revient, pour conclure, sur la responsabilité de ceux qui « sont sur les lieux et ont une expérience journalière de tout ce qui est nécessaire pour la commodité

1. "Mémoire sur le règlement à faire pour la police générale des arsenaux de Marine ", in Pierre Clément, Lettres de Colbert, Paris, 1868, t. III, p. 224.

2. Cf., par ex., ibid., p. 224 : " Instructions pour le Sr Matharel Conseiller du roy en ses conseils, que S.M. envoye au port de Toulon pour y faire les fonctions d'Intendant ", avril 1670.

3. Ibid., p. 287 : « Mémoire sur le règlement à faire pour la police générale des arsenaux de Marine ", oct. 1670.

4. Ibid., p. 296: " Mémoire sur tout ce qui doit s'observer pour former les magasins des arsenaux de la Marine du Roy $», 8$ oct. 1670. 
de tant de différentes fonctions "; il leur demande de "corriger, ajouter ou diminuer pour parvenir à la perfection de cet établissement $"$ s, un arsenal.

Concevoir un tout, non comme un ensemble de parties combinables, mais comme une combinaison de fonctions différenciées et hiérarchisées, est une vision proprement technique d'une organisation. Jusqu'à quel détail Colbert pouvait-il en faire usage, il est bien difficile d'en juger. On remarquera seulement que le pragmatisme qu'on lui reconnaît habjtuellement pourrait être reconsidéré sous cet éclairage ; en particulier, le rôle et la signification des enquêtes et expériences qu'il conduisit. Les pages qui suivent exposent l'une de ces "expériences ». Elle fut exceptionnelle à la fois par l'importance des moyens mis en ouvre, et par le travail de réflexion sur l'organisation de la production de vaisseaux qu'elle dut mobiliser.

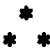

En juillet 1678, Colbert fit à l'intendant de Toulon, Arnoul, une étrange proposition : faire bâtir un vaisseau de 26 à 30 pièces de canon en un ou deux jours de temps ${ }^{6}$. L'occasion en devait être le voyage projeté du roi dans ce port ; l'ambition, de lui fournir « la plus grande satisfaction possible ». Le projet devait rester secret, "le principal consistant à l'exécuter sans qu'on s'y attende ». L'effet de surprise ne fut pas atteint et le roi, averti, ne vint pas. Toutefois, pendant un an et demi, dans les trois arsenaux de Rochefort, Brest et Toulon, on se prépara?.

L'intention politique était claire : le projet s'inscrivait dans un ensemble de dispositions qui visaient à « affectionner de plus en plus

5. Ibid., p. 297.

6. Archives nationales, Marine B2 39 (377). Des 1671, Colbert en avait conçu le projet. Le 2 janvier 1671, il ecrivait à Matharel, Intendant du Port de Toulon de « travailler préparer toutes les pièces de bois qui entrent dans la construction d'un vaisseau, afin que, lorsque le Roi ira à Toulon, [...] vous puissiez faire commencer et achever un vaisseau en sa présence, pendant les 10 ou 12 jours qu'il y sera ", in P. CLÉmENT, op. cit. supra n. 1, p. 327. Matharel s'y prépara, mais le projet fut momentanément abandonné.

7. On trouve aux seules Archives nationales, une trentaine de lettres où il est question de ces " vaisseaux basty devant le Roy ». Elles se trouvent dans les séries Marine B2, B3 et D1 (citées par la suite comme B2, B3 et D1). L'idée de ces préfabrications était peut-être inspirte de ce qui se pratiquait pour la construction de galères. Le 16 mars 1669, Colbert ecrivait a Arnoul père, Intendant de Marseille, pour lui demander de se pretparer à faire construire de nouvelles galères et ajoutait : « prenez garde seulement que vous en ayez toujours une en paquet et que vos maistres de hache soyent en pratique de la bastir et mettre en mer en vingt quatre heures s'il est possible ", in P. CLÉmENT, op. cit. supra n. 1, p. 107. 
le roi à sa Marine ", c'est-à-dire - et là résidait la difficulté - " lui inspirer pour l'armée de mer une affection comparable à celle qu'il portait à l'armée de terre ${ }^{8}$.

La première de ces dispositions consista à orner le cabinet du roi de peintures et dessins « représentant des tableaux de marine ". Puget et Jean-Baptiste la Rose y travaillèrent. Cependant, ces " peintures froides " ne suffisant pas a exciter l'imagination du roi, "Colbert persuada à Louis XIV de faire construire de petits navires et de composer ainsi une flottille de bâtiments en miniature, décoration charmante pour le canal de Versailles, et gracieuses embarcations pouvant servir aux promenades de la Cour sur cette vaste pièce d'eau " 9 . Le roi accepta, Colbert s'activa. En janvier 1668, il écrivit à l'amiral Beaufort : « Vous commanderez, s'il vous plaît à Dumas de faire faire, sans perte de temps, deux petits bastimens comme je le luy ay demandé pour le canal de Versailles, le Roy les désirant incessamment " ${ }^{10}$. La Marine de Versailles ne cessa de s'augmenter, ces ébats nautiques devenant l'occasion de montrer à la Cour les progrès de l'architecture navale ". Cependant, Colbert continuait à inciter le roi à aller voir les choses au vrai dans ses ports. Lorsqu'il obtint la fallacieuse promesse de cette visite, il imagina la mise en scène qui nous occupe.

Il y avait un précédent : la construction d'une galère en un jour, à Venise, à l'occasion des grandes fêtes données au roi Henri III en 1574 '2. On sait qu'à Venise l'effort d'organisation du travail et de rationalisation de la construction navale fut, dés le XVie siecle, l'enjeu de batailles politiques et économiques. En 1530, Vettor Fausto y avait conçu

8. Auguste JAL, Abraham Du Quesne et la marine de son temps, Paris, 1873, t. II, p. 45. Jal ne fait que quelques allusions dispersées à ce projet de constructions préfabriquées.

9. Ibid., t. 1, p. 539.

10. B2 34 .

11. Jusqu' la mort du roi, on entretint a Versailles, sous les ordres du marquis de Langeron, Inspecteur des constructions navales, quatre charpentiers, un calfat, un maîtrecanonnier, huit matelots, un capitaine de l'escadrille et un lieutenant commandant sous ses ordres. Pour parfaire la flotte, on y joignit une galère construite par Chabert et couverte de sculptures par Philippe Calfieri et Briquet. Enfin, quatre gondoliers furent amenes de Venise. Pour plus de détails, cf. A. JAL, Dictionnaire critique de biographie et d'histoire, Paris, 1864, article "Versailles ".

12. Dans son « Mémoire sur le réglement faire pour la police générale des arsenaux de la Marine n, in P. Clément, op. cit. supra n. 1, p. 295, Colbert demande aux intendants, pour " contribuet à construire, équiper et armer les vaisseaux avec facilité et en peu de temps ", de prendre pour modèles " à l'égard des galères, les Vénitiens, qui firent bastir une galère en vingt-quatre heures de temps en presence de Henri III, et, a l'égard des vaisseaux, les Hollandois, dont les habitants de Sardam offrirent, pendant leur derniere guerre contre l'Angleterre, qu'en leur donnant trois mois d'avance, ils fourniroient, apres ce temps là expiré, tous les jours un vaisseau pour mettre en mer ". 
la fameuse galère à cinq rangs, imité des Anciens ${ }^{13}$. Dans un contexte fort différent de la France de Colbert, il avait eu le même projet de maîtrise "scientifique " de l'art de la construction navale. Si ses ambitions humanistes furent empêchées, en particulier par les réticences de corps de métiers, il reste que l'idée d'un tel spectacle offert au nouveau roi de France en porte la marque ; même si c'est pour beaucoup un témoignage du goût que l'on avait pour les grandes manceuvres bien réglées.

Car le modèle était militaire : manifestation par excellence de la puissance royale. Non pas une parade, mais une " action ", avec ceci de particulier qu'il s'agissait d'une entreprise concertée de production, et non de destruction; d'un exploit dont les héros n'étaient pas des officiers et des soldats, mais un Intendant et des ouvriers. Voilà qui confirmait spectaculairement un thème récurrent à l'époque et particulièrement cher à Colbert : celui de la grandeur que confèrent à un pays le développement et la bonne réglementation de sa production; partant, l'augmentation de sa richesse par une meilleure productivité du travail ${ }^{14}$. Produire ses propres vaisseaux était, en bon mercantilisme, une entreprise contre la puissance des nations ennemies ${ }^{15}$, d'autant que c'était produire un instrument de guerre. Dans cette promptitude à en réaliser un exemplaire, le roi devait voir la preuve de la capacité de sa Marine à se préparer pour de futures victoires, et sans doute était-ce un motif considérable du contentement que l'on voulut faire naitre en lui. L'écrivain de Toulon, chargé de relater l'épreuve de montage qui y fut faite le 14 juillet 1679 , y insista :

" L'on estoit desja convaincu, écrit-il, par tous les combats et exploits de ses armées nouvelles que la Marine estoit à present en France dans un plus haut degré de perfection que dans aucun autre royaume. Monseigneur

13. Cf. Ennio Concina, "La fascination de l'antique en fait d'architecture navale ", Amphion, 1, 1987, p. 21-26.

14. Ce qui apparaît assez peu dans les doctrines économiques, toutes occuptes par la question de l'argent et du taux d'intérét, est néanmoins l'objet de réflexions constamment reprises à propos du travail par entreprise, en régie ou à l'ćconomie, sur l'emploi des ouvriers à la journée du roi ou à la tåche. A partir du xvil' siécle, les ministres de la Guerre et de la Marine demanderont que, dans les Places et dans les Ports, on établisse des tableaux comparés de la dépense selon ces différents modes de production. Calculs qui étaient compliqués par la diversité des situations des ouvriers. La présence des galériens et, a partir de 1748, des bagnards, dans les arsenaux de la Marine, et leur mise au travail, d'abord ponctuelle, puis systématisée dans la première moitié du XIX' siécle, renouvelle le débat sur les avantages respectifs des différents modes de rétribution et d'organisation du travail.

15. Ceci double titre, puisqu'on pourra mieux les combattre et qu'on ne leur achetera pas de vaisseaux, ce qui faisait sortir l'argent du royaume, au profit de l'ennemi. Pourtant, l'on continuera à passer des marchés et à faire construire à l'étranger. 
Colbert et Mr le Marquis de Seignelay en ont voulú donner tout nouvellement une preuve bien convaincante, et sur laquelle on peut concevoir une grande idée de l'ordre admirable qu'ils ont mis dans les Ports $"{ }^{16 .}$

Cette action si extraordinaire était également la preuve du soin que prenaient Colbert et Seignelay

" de choisir de bons subjects pour en avoir la direction, de les eslever eux mesmes dans leur jeunesse, et de leur inspirer un esprit d'ordre, de prevoiance et d'œconomie avec le zèle et l'application qu'ils doivent avoir pour estre capables de bien exécuter leurs ordres et leurs desseins $" 17$.

Dans l'organisation de la Marine que Colbert avait mise en place, l'Intendant, de même que l'Inspecteur des constructions, avait pour tâche à la fois d'assurer la réalisation des décisions du Ministère et d'étudier les habitudes et savoir-faire locaux dans leurs particularités, et même leurs singularités, pour les faire connaître à la Cour ; ceci, à l'aide de mémoires, de tableaux qu'ils devaient compléter, de réponses à des questionnaires. Transmission de l'information dont la rigueur méthodique apparentait l'administration à une sorte d'organisme de recherche scientifique et technique, l'Intendant jouant un rôle décisif dans le double mouvement institué de déduction des principes jusqu'à leur réalisation pratique, et $d$ 'induction des réalisations particulières vers des principes plus généraux ; il devait rendre ainsi possible la confrontation, à l'échelle du royaume, des savoirs locaux et donc contribuer à l'uniformisation de pratiques hétérogènes ${ }^{18}$. L'affaire des vaisseaux bâtis devant le roi obéissait à ce système général. Dans sa première lettre à Arnoul, en juillet 1678, Colbert affirmait :

" Je suis persuadé que pourveu que vous vous mettiez dans la teste de reussir à ce que je vous propose, vous y parviendrez aisément. Cette proposition vous paroistra peut estre extraordinaire, mais en une affaire comme celle-là, il ne faut pas vous laisser rebuter par les premières difficultés $" 19$.

16. D1 15 (4).

17. Ibid.

18. La mise en place des tcoles de construction et d'hydrographic dans les ports en est l'expression la plus évidente. L'un des rôles de l'Inspecteur et de l'Intendant était de rendre explicite le savoir "muet " des maîtres de metiers; d'en produire le discours, et, ce faisant, de l'inclure dans un enseignement uniforme et controlable par le Ministère.

19. D1 15 (4). 
Devant une situation inédite, il convenait de partir, non pas de la multiplicité des tâches à accomplir, des problèmes pratiques à résoudre, de la complexité des opérations à combiner, mais de l'idée : se la mettre en tête. Admettre a priori que cette proposition extraordinaire n'était pas irréalisable, disposer son esprit à " l'affaire ". Il devait suffire de bien en concevoir l'idée, le principe, pour dépasser le premier mouvement, celui que dictait un trop grand attachement à la coutume : rejeter cette " nouvelleté ». Adhérer à l'idée, la faire sienne. L'affirmation de la puissance du projet, pourvu qu'il soit bien concerté, l'emporte sur une vision trop pragmatiste qui invite à la répétition. Il y a du panache dans une telle confiance en l'idée. S'y refuser d'emblée c'est demeurer " rez pied rez terre ", à l'instar d'un ouvrier. C'est aussi refuser de servir, car cette idée est un ordre. S'y conformer en conscience est pour l'Intendant tenir son emploi, comme on dit " tenir son rang " : réaliser l'ordre établi. Quitte ensuite à « mettre la main à la conscience ", selon l'expression consacrée : prendre la mesure de l'idée en la confrontant aux dispositions que sa réalisation suppose. Ces allers et retours de l'idée à la pratique, et de la pratique de la chose à l'idée, s'ils correspondent à une philosophie de l'action, prennent la tournure d'une véritable rhétorique administrative : les lettres de la Cour aux Ports, à l'occasion de notre affaire y obéissent.

Quant aux réponses des Ports, elles développaient un certain nombre de thèmes repérables : lors même que des réticences ou des refus s'y exprimaient, ces revendications obéissaient à l'ordre général mis en place par Colbert ; c'est dire que les Intendants mettaient en avant les situations concrètes liées à des particularismes locaux, des difficultés temporaires; en gros, ils argumentaient à partir des contraintes matérielles et singulières auxquelles ils devaient faire face sous l'impulsion des ordres venus de la Cour ; la mise en évidence des différences locales et la fréquente affirmation de leur "nécessité " étant, bien sûr, l'effet de la volonté d'uniformisation. Dans ces conditions, contrôler depuis le Ministère les ajustements de la pratique aux règles énoncées, supposait que l'on prît la mesure des marges de liberté nécessaires pour que quelque chose se passât. Il convenait de savoir à partir de quel point réglementer devenait inoperant, ce qui est tout autre chose que d'accepter l'approximation, l'ambition demeurant de réduire ces marges : ces manquements à la règle n'étaient tolérés qu'à la condition de faire l'objet d'études attentives à en supprimer les inadvertances. La correspondance entre la Cour et les Ports nous apprend que c'est en portant une attention soutenue aux procédés d'organisation du travail et de fabrication, c'est en visant à maîtriser le processus de réalisation des choses par le biais d'une instruction de plus en plus « technique » que, dès Colbert, 
la normalisation des vaisseaux est visée. Il ne s'agit pas seulement d'évaluer des résultats, mais des systèmes de construction, à une époque où les charpentiers sont les man̂tres des chantiers. Il ne faut donc pas s'étonner s'il fallut un siècle pour qu'effectivement le contrôle ne portât plus tant sur l'objet à réaliser : le navire, que sur les méthodes choisies pour réaliser " l'application " des principes de l'architecture navale.

Telle était cependant la représentation idéale de la perfection administrative qui présidait aux enquêtes, résolutions, ordonnances dont le Ministère accablait les Intendants. Même si cet idéal n'était qu'à l'horizon des efforts entrepris pour y parvenir, il était également à poursuivre. Les tentatives d'uniformisation des mesures, la substitution d'un enseignement à l'apprentissage traditionnel, la surveillance des chantiers par les Intendants et les Inspecteurs, le développement des sciences physiques du navire, toutes ces dispositions étaient autant de jalons à partir desquels on devait persévérer dans l'effort de rationalisation de la construction. Si l'on admet que l'anticipation des effets de l'action, au service de leur évaluation préalable, est l'objectif principal de l'administration, pouvait-on alors imaginer philosophie plus adéquate? On comprend aussi que l'affaire des vaisseaux bâtis devant le roi pouvait être à la fois la mise à l'épreuve de ce qui était, et le symbole de ce qui devait être.

Mise à l'epreuve, d'abord, de la maîtrise technique de la construction. Car il s'agissait d'un ordonnancement réglé d'opérations dont on voyait la production ; non pas un ordre des choses, mais un ordre de l'action. Partant, c'etait le contrôle du Corps de la Marine qui devenait manifeste à travers la discipline des actes dans l'espace et le temps. La stricte programmation de l'ouvrage montrait jusqu'à quel point l'on détenait la capacité de tout prévoir : cet ordre était admirable parce qu'il était préconçu. Situation limite et critique : l'organisation était confrontce à son idéal. Or l'idéal ne pouvait devenir rél, au point d'imperfection où l'on était encore, que si les conditions rassemblées étaient extraordinaires. Cette parade symbolique de la perfection viste, livrbe au regard du roi, était également un dispositif pour en obtenir les moyens. La preuve la plus patente est, qu'en l'affaire, on ne regarda pas à la dépense. Certes, Colbert exigea une estimation des épreuves : combien faudra-t-il d'ouvriers ? combien en coutera-t-il chaque fois ? mais c'etait pour préciser aux Intendants : " Parce que si vous croyez que cela puisse contribuer à accoustumer les ouvriers à cette diligence, il faudroit sans difficulté en faire faire plus d'une espreuve $"{ }^{20}$. On ne fera pas de

20. "Lettre de la Cour aux Intendants de la Marine ", Saint-Germain, le 10 sept. 1678, in $\mathrm{B2} 39$ (637). 
difficulté à débourser pour accélérer le travail ; l'essentiel étant d'obtenir une réussite si éclatante, que le roi pourrait décider l'augmentation des finances de la Marine. Dans la stratégie de Colbert, c'est un « grand coup " où la dépense ne peut être calculée à l'ordinaire : on en espère un gain considérable.

Puisque le roi ne vint pas, le projet de ce grand spectacle fut un échec. Augustin Jal, historien de la Marine, essaya de le sauver en avançant l'idée que

" tout le temps passé aux répétitions ne fut point perdu pour les ouvriers ; ils y apprirent à se hâter sans se gêner mutuellement, à se bien disposer pour que chaque groupe agît avec ensemble ; enfin, à se servir au mieux des apparaux propres à manier les membrures, les baux, les grosses courbes et le reste ; les matelots y apprirent à gréer rapidement un navire, sans que la rapidité nuisît au bon établissement de toutes les manceuvres dormantes et courantes ; pour tout le monde ce fut un témoignage de ce que peut la volonté servie par l'intelligence $" 21$.

Cet optimisme de bon ton paraît raisonnable. Ce n'est jamais qu'une vue de l'esprit. Les documents dont nous disposons nous permettent d'aller plus avant dans l'examen des effets de cette longue et dispendieuse préparation. Pendant cette période, et à cette occasion, que se passa-t-il dans les Ports, entre la Cour et les Ports ?

Dès sa première lettre, Colbert énumérait les conditions pratiques de la réussite du projet. Elles concernaient les trois facteurs mis en cuvre : matières, espace, temps. "Je suis persuadé, écrivait-il, qu'en faisant façonner tous les bois à l'advance, mettant les membres en estat d'estre mis en place et ayant tout le nombre d'habiles ouvriers nécessaires pour la promptitude de ce travail, on pourroit donner cette satisfaction à Sa Majesté " ${ }^{22}$. La combinaison habituelle de ces facteurs devait être reconsidéree, du fait que la promptitude commandait absolument. La méthode de montage en était nécessairement modifiée. Les autres recommandations qui, classiquement, étaient directives : solidité, économie, durée, devenaient secondaires, se modifiaient, ou même disparaissaient des consignes de la Cour - nous l'avons vu pour la dépense -, ce que l'on supporta mal dans les Ports. Piqués au jeu, après quelques réticences et atermoiements, Intendants et Maîtres-charpentiers auraient voulu en faire une méthode de construction reconnue, que l'on pat mettre en cuvre pour produire des vaisseaux et non pas seulement du spectacle :

21. Op. cit. supra n. 8, t. II, p. 42.

22. "Lettre d̀ Arnoul ", Saint-Germain, le 26 juil. 1678, in B2 39 (377). 
une véritable « action » et non une simple parade. C'est l'une des divergences qui, très vite, apparurent entre le Ministère et les Ports. En quelque sorte, tout en adhérant au projet de Colbert, les Intendants le subvertissaient, prétendant ainsi continuer à obéir aux impératifs qui devaient, a l'ordinaire, régler la bonne gestion de la construction. Colbert prit fort mal cette dialectique subtile qui s'exprima particulièrement à Toulon. Pour en goûter toute la saveur, il faut suivre l'affaire dans le détail. Nous le ferons en examinant successivement les conditions de la mise en œuvre des trois facteurs évoqués plus haut : matières, espace, temps.

\section{LES MATIËRES}

« Appliquez vous toujours, écrit Colbert, à choisir les plus belles pièces de bois et les mettre à part, et faites les chercher partout [...]. Observez, autant qu'il vous sera possible de faire tous les membres d'une seule pièce de bois [...] et s'il est impossible, faites en sorte de les faire de moins de pièces qu'il se pourra $\gg 23$.

Voilà déjà qui n'était pas ordinaire. La difficulté à trouver des bois de grandeur suffisante pour les pièces les plus importantes du corps du vaisseau - la quille, l'étrave et l'étambot, mais aussi les couples - était habituellement surmontée selon deux procédés : le Ministère, ayant décidé la construction d'un certain nombre de vaisseaux de rangs déterminés, en envoyait les mesures générales aux Ports. Elles devaient être respectées, ce qui en principe assurait l'uniformité des vaisseaux de la flotte. Les charpentiers examinaient alors les bois dont ils disposaient et, souvent, la nécessité l'emportant sur le respect des proportions définies par le Ministère, les modifiaient. L'autre solution, lorsqu'on était à court de bois suffisants, consistait à recourir aux assemblages : les membres étaient composés de trois, quatre, cinq pièces quelquefois ${ }^{24}$. L'ingéniosité des solutions se perfectionnait grâce à l'examen de pièces ainsi fabriquées, et qui avaient

23. "Lettre à de Seuil \#, Fontainebleau, le 6 sept. 1678, in B2 39 (449).

24. Cf. le « Proçs-verbal du Conseil de construction du Port de Toulon réuni le 5 mars 1690", in Archives du Port de Toulon, 3 Al 1 : "Ce jourd'huy [...] le Conseil de Construction estant assemblé pour examiner les propositions données par le Sr Coulomb, Maistre charpentier sur la construction de deux fregattes [...] que le Roy a ordonné [...] il sera fait en sorte que la hauteur dudit bastiment soit composte de cinq pizces affin qu'on puisse plus facilement (trouver) de bois dans l'arsenal pour utiliser le vieux. 》 
servi ${ }^{25}$. Dans l'épreuve du vaisseau bâti devant le roi, il convenait de ne pas multiplier ces assemblages « pour la promptitude ", tout en respectant cependant le principe de cette exhibition : « vous devez faire estat de monter ce vaisseau de toutes ses pièces en présence de Sa Majesté et non de monter auparavant la quille, l'estrave et l'étambot », rappelle Colbert à de Seuil ${ }^{26}$ qui proposait de procéder à des montages préalables de sous-ensembles ${ }^{27}$. Il n'était pas question de transiger là-dessus. De Seuil le comprit si bien que, relatant l'épreuve faite à Brest le 4 août, il signala que " mesme, la quille [fut] renversée le dessus dessous " ${ }^{28}$ : on ne pouvait mieux voir les membres démontés. Encore convenait-il qu'ils fussent de belle apparence : « surtout prenez bien garde, enjoint Colbert, que toutes les pièces paroissent passées au rabot ${ }^{29}$ : il suffirait qu'elles le paraissent pour le spectacle. Toutefois, on précise tout de suite que ces pièces devront être rabotées en effet, si de Seuil l'estime nécessaire. Nécessaire à quoi ? À l'esthétique, ou à la précision des ajustements sans lesquels ils ne pourraient se faire promptement ? Au fil des correspondances, le souci d'augmenter " la propreté du bastiment 》 l'emporte : on devra « passer au rabot pour la justesse " ${ }^{30}$.

Il est un autre problème, propre aux matières, qui se pose dans tous les cas de préfabrication : celui du maintien en état d'être montées des pièces façonnées, de leur stockage. Il ne semble pas que le problème ait été directement abordé avant les premières épreuves. C'est en réponse aux remarques de Denuin dans le compte rendu du montage effectué le 25 mai 1679, que Colbert donna son avis : il faudrait « mettre toutes les pièces sous l'eau et les conserver pour les assembler lorsque Sa Majesté ira à Rochefort " ${ }^{31}$. À Brest, on envisageait de les conserver à l'air libre. Toutefois, de Seuil précisa qu'« il n'y a point à Brest de magasin ny de hangard pour serrer les bois de cette fregatte " ${ }^{32}$. Ces deux modes de conservation des bois supposaient, les pièces étant façonnées et devant être constamment disponibles, des précautions et donc des aménagements

25. Colbert mit très minutieusement en place les conditions pratiques de ces examens qui portaient sur l'état de l'ensemble du vaisseau au moment des désarmements : des officiers de marine, assistés de charpentiers, devaient y procéder. Leurs remarques étaient enregistrées et consignées selon des " devis ». Joints aux devis de constructions et aux journaux de bord, ils constituaient un ensemble de documents susceptibles d'être utilisés lors de la conception de nouveaux modèles. L'ambition étant de convertir le simple usage en " expérience ".

26. Lettre du 6 sept. 1678 , in B2 39 (449).

27. Lettre du 2 sept. 1678, in B3 32 (29).

28. DI 15 (3).

29. Le 6 sept. 1678 , in B2 39 (449).

30. Lettre de la Cour du 26 dec. 1678, in B2 39 (637).

31. Lettre du 4 juin 1679, in B2 41 (309).

32. Relation du 4 août 1679 , in D1 15 (3). 
inconnus à l'époque. En outre, leur maintien en état d'être rigoureusement ajustées, excluait de trop nombreux montages, démontages, remontages des pièces, qui les endommageaient, cependant qu'il était indispensable de procéder à plusieurs épreuves pour régler la programmation du travail. Là-dessus, une polémique s'engagea entre les Ports et le Ministère.

À Rochefort, on commença par annoncer qu'au cas où le roi ne viendrait pas, « il faudroit faire frapper les cloux de laditte fregatte [...] la calfater et la mettre à l'eau, afin qu'elle ne s'endommage pas ». Puis, l'on signala qu'ayant " desja esté faite deux fois, il est impossible que les cloux ne gastent un peu les membres ". Au passage on remarquait que " c'est une des plus belles fregattes qu'on ait veue " : la gâter par une autre épreuve serait aberrant, il vaudrait mieux la conserver au roi. La proposition qui suivait allait de soi : " supposé que le Roy vint à Rochefort l'annee prochaine, [Denuin] en feroit faire une autre de mesme gabarit, et par les mesmes charpentiers " ${ }^{33}$.

Un savoir-faire était acquis : on ne saurait avoir de surprise, alors que la matière se détériore, que les formes se déjettent sous l'action du temps. La confiance, dans les Ports, va plus aux hommes qu'aux choses. Et puis, on respecte une production comme une ceuvre, on répugne à défaire ce qui a été fait. Alors que Colbert insiste pour avoir la frégate toute disposée au montage, Denuin vante longuement sa beauté et " prie d'accorder quelque chose aux deux charpentiers qui l'ont bastie nommés Honoré Mallet et l'autre Me Pierre Mallet son fils. Ce dernier est jeune et trés habile et deviendra le meilleur charpentier du Port pour la construction $"{ }^{34}$. On s'inquiète ainsi de l'avenir de l'arsenal.

On voulait en cette action, tout assujettir à la promptitude. L'idée d'une préfabrication achoppait non seulement aux modifications de matières rebelles et l'inexistence des aménagements nécessaires pour les conserver, mais également à des conceptions du rapport de la production au produit et du produit à son usage, en général d'autant moins conciliables qu'elles ne sont jamais complètement explicites ${ }^{33}$.

Il est un dernier point concernant les matières en même temps que les méthodes d'assemblage, qui fit l'objet de discussions avec Colbert : celui des pièces qui devaient maintenir les parties ajustées; si, pour

33. D1 15 (1).

34. Ibid.

35. La préfabrication de pièces identiques et donc interchangeables pour chaque rang de vaisseaux, et leurs modalités de magasinage, fut toujours source de conflits entre administrateurs de l'Arsenal et officiers de Marine. Cf., par ex., le « Proces-verbal du Conseil de construction du Port de Toulon réuni le 3 mars 1834 " sur l'aménagement du magasin des mâtures, in Archives du Port de Toulon, 3 Al 30. 
l'essentiel, celles-ci étaient chevillées de bois, on clouait néanmoins avant de calfater. Sur les conseils de Colbert, lors des dernières épreuves à Rochefort et à Brest, on se contenta de cheviller pour éviter les détériorations. La même recommandation fut faite à Arnoul qui affirmait que le démontage et remontage d'un même vaisseau était impossible " parce qu'il faut clouer à chaque fois, et qu'il faut le clouer non seulement parce qu'il ne pourroit se soustenir autrement [...] et estant cloué une fois, il ne pourroit plus se démonter $"{ }^{36}$. La difficulté était particulière à Toulon, et Arnoul se fit le porte-parole des ouvriers pour le préciser : « Comme ils n'ont pas neantmoins icy l'usage des chevilles de bois, et qu'ils croyent qu'ils auroient de la peine à les repousser, ils ne jugent pas pouvoir s'en servir utilement non plus que des cloux pour la mesme difficulté à cause qu'ils sont pointus. " L'Intendant proposa alors un compromis : " Je feray faire des petites chevilles de fer de la longueur mesme des cloux desquels elles ne differeront que parce qu'elles ne seront pas pointues, et de cette manière ils en viendront à bout " ${ }^{37}$. Cependant, on n'était pas au bout de la correspondance entre la Cour et Toulon sur cette question. Colbert, agacé, n'admit pas cette particularité toulonnaise et, le 24 mars, Arnoul, qui multipliait les marques de bonne volonté, dut répéter :

"Si les charpentiers font difficulté de se servir de chevilles de bois, c'est qu'ils ne sont pas accoustumés de s'en servir en ce pays comme en Angleterre et en Ponant et qu'en les poussant et repoussant ils pourroient causer de l'embarras que l'on doit éviter autant qu'on peut dans une occasion pareille. "

Il réitéra alors sa proposition, signalant que la chose avait été discutée du temps de l'Intendant qui l'avait précédé, et que « sur les Mémoires que l'on envoya, Sa Majesté trouva bon que l'on n'employa icy que du fer suivant leur coustume ${ }^{38}$.

Cependant, c'est à propos de l'organisation de l'espace : chantier et ateliers qu'Arnoul se fit le plus exigeant.

36. Lettre du 17 janv. 1679, in B3 32 (40).

37. Lettre du $24 \mathrm{fev} .1679$, in ibid.

38. Lettre du 24 mars 1679, in B3 32 (212). La technique de chevillage de bois est empruntée aux Anglais. En 1670, Colbert envoie l'un de ses charpentiers, Hubac fils, en Angleterre, en lui enjoignant de « vous rendre savant sur l'usage des chevilles de chesne vert et des lieux où ce bois se prend et comment on le goupille ", in P. CLEMENT, op. cit. supra n. 1, p. 234. 


\section{L'ESPACE}

Des trois Intendants engagés dans ce projet, Arnoul fut sans conteste le plus réticent. Le 17 janvier 1679 seulement, il donna son avis : une telle diligence était impossible, sauf à accélérer le travail par le moyen d'une halle et d'une forme. Une halle, afin de pouvoir transporter rapidement les grosses pièces au-dessus du chantier. On envisageait bien à Marseille de monter une galère dans des conditions de promptitude comparable, mais un vaisseau n'est pas une galère dont toutes les pièces peuvent se déplacer à bras d'homme. Une forme, pour que le navire puisse être mâté et agréé sur place. Pour appuyer ce point de vue, Arnoul requit les deux principaux Maîtres-charpentiers du Port, L. Coulomb et Chapelle, faisant écrire à chacun une lettre. Coulomb affirma qu'« il seroit impossible de pouvoir remonter le susdit vaisseau dans la place où il est sans forme ny hale en moins d'un mois, quel nombre d'ouvriers que l'on y mette veu mesme que la trop grande quantité pourroit embarasser ". Quant à Chapelle, il comptait vingt jours sans forme ni halle, huit si l'on en disposait « de la manière qui nous a esté demontrée par M. l'Intendant $"{ }^{39}$.

Ce coup monté déplut souverainement à Colbert qui fit une réponse d'une rare violence, glissant au passage une pointe méchamment spirituelle :

« Si vous aviez fait comme les autres intendants, vous auriez un peu plus examiné la matière et vous n'auriez pas tellement appliqué votre esprit à chercher les difficultez qu'il ne vous en fust resté un peu pour trouver le moyen de les surmonter $" 40$.

Qu'Arnoul fasse écrire à des charpentiers ces " certificats signés d'eux " pour s'opposer à l'idée conçue à la Cour et favoriser la routine locale, voilà qui ne convenait guère aux exigences de son emploi. Cependant, on peut penser qu'Arnoul voulait tout simplement tirer parti de l'édification de ce vaisseau pour obtenir la forme et la halle qu'il convoitait : occasion privilégiée, puisqu'on ne regardait pas à la dépense.

La forme ne fut pas achevée à temps. Sa conception, œuvre de l'ingénieur Niquet, ne manquait pas d'ingéniosité. On parlerait plus volontiers d'audace inventive que d'esprit de routine. Dès

39. B2 32 (42).

40. Saint-Germain, le 3 févr. 1679, in B2 41 (68). 
janvier 1679, le modèle était établi et un mémoire envoyé à la Cour.

C'était une forme de bois flottante, qui pouvait être fermée et vidée au moyen d'une vis d'Archimède. Dans sa lettre du 17 janvier 1679, Arnoul explique que

« quand je ne serois pas aussi asseuré qu'il me semble que je le doibts estre qu'elle reussira, ce seroit une espreuve necessaire a faire veu que depuis que je connois ce terrain, et la quantité de sources qui en sortent, je ne croy pas qu'on pust venir à bout d'en faire autrement en quelqu'endroit que ce soit ${ }^{41}$.

C'est du désespoir à pouvoir fonder qu'était née l'idée de cette forme de bois. Arnoul voulait qu'elle restât à flot. Il envoya un devis à Colbert qui objecta que le fond n'en serait pas assez solide pour porter une masse comme celle d'un vaisseau, et qu'il fallait la couler quelque part dans le port. Arnoul, encouragé par Vauban qui avait approuvé ce système, répondit en proposant qu'on renforçât le fond. Colbert, qui n'était pas convaincu, demanda le 28 février à Duquesne qui était à Toulon, un examen et une décision. Duquesne confirmant les appréhensions de Colbert, la forme fut effectivement coulée en 1680 mais ne servit jamais. En 1685, elle fut troquée contre quelques clous et ferrures ${ }^{42}$.

Si ce projet de forme se révéla à l'épreuve plus hardi que bien concerté, il restait la halle dont l'édification et l'aménagement permirent, en effet, de monter le vaisseau avec une promptitude qu'on n'atteignit pas dans les autres ports. On eut d'abord quelques doutes sur son efficacité, et l'écrivain chargé de relater la première épreuve complète de montage, du 14 juillet 1679, affirma que l'on fut surpris. " Le nombre de plus de deux mil cordages et de cinq cent poulies " qu'on y voyait « paroissoit à plusieurs une confusion qui fesoit craindre un embaras dans le denouement et l'execution ". D'autant que " cette mesme halle et tous les echaffaults qui estoient autour servoient à renfermer toutes les pièces necessaires à cette construction ". Cependant, leur accord et arrangement étaient tels que tout "s'est

41. B3 $32(40)$.

42. Le 20 août 1685 , Vauvré, successeur d'Arnoul, mit en adjudication cette " grande caisse " qui " se trouve inutile au service de Sa Majesté, attendue qu'elle est en partie pourrie ». Le 6 septembre, François Julien obtint le marché pour 6350 L., " pour le montant duquel il s'oblige à fournir $[\ldots]$ des cloux au poids, ferrures de gouvernails, de chaloupes... ", in Archives du Port de Toulon, 5E 179 (100). 
pourtant developpé d'une manière si facile et surprenante qu'elle servira dans la suite de modele en semblable cas $"{ }^{43}$.

Sur cet arrangement, l'écrivain ne s'attarda pas, signalant seulement que « les peintres qui l'ont voulu dessigner n'ont jamais pu en venir à bout et les Mes charpentiers qui ont rangé toutes choses disent eux-mesmes qu'ils ne pourroient en donner qu'une legère idée ". Cependant, nous apprenons que du Maitz, Commissaire de marine à Toulon, « a fait sur cet arrangement seul, une relation toute entière qu'il a envoyée dans les autres ports ». Nous n'avons pas ce document ; par contre, le Mémoire de ce qui fut observé à Brest, le 4 août, nous fournit quelques détails sur la manière dont on aménagea l'espace de travail autour du chantier : la conception en fut toute autre qu'à Toulon.

Tous les membres, baux et bordages, marqués par chiffre, furent rangés le long du quai des constructions dans l'espace de plus de deux cents pas, dont la pièce la plus proche du chantier était éloignée de dix pas. Comme on ne disposait pas de halle,

« pour le soulagement des ouvriers et facilité à mettre en place toutes les pièces de bois de la construction dudict vaisseau, a esté estably dix petits masts debout de chaque costé du vaisseau esgalement distans l'un de l'autre au bout desquels il y a un bout d'un petit mast plact, en forme d'une fleche de grue ou arboutant tournant autour dudit mast pour pouvoir prendre les membres à six pieds de distance autour de luy, par le moyen d'un palan frapé au haut du bout du petit mast $" 4$.

Cette enfilade de grues tournantes ne devait pas manquer d'allure. Il semble qu'à Rochefort elles aient été inventées pour la circonstance, et il serait intéressant de savoir si l'appareil fut perfectionné. Si l'on y joint le modele de halle avec ses aménagements, envoyé de Toulon dans les autres ports pour servir dans d'autres cas semblables, on pourrait penser avec Jal que, tout compte fait, ces épreuves ne furent pas complètement inutiles. Cependant, Jal retint pour l'essentiel leurs effets sur la

43. D1 15 (4). Pour persuader le Ministère de l'opportunité de ces constructions, Arnoul remarque que « l'on ne peut trouver meilleure conjoncture qu'd présent, puisque défaisant le Leger et la Normande on se sert de tous les baux et de toutes les courbes et tous les fers qui s'en retirera ce qui ne pourra pas se retrouver dans un autre temps, en sorte qu'il en couteroit une fois autant " (Lettre du 17 janvier 1679). On utilisait autant que possible les bois et fers retirés de la démolition des navires pour construire les batiments de l'arsenal, en particulier pour les charpentes et couvertures. On peut se demander si cette pratique, et les astuces de charpenterie qu'elle exigeait n'a pas joué un role dans la couverture de grandes surfaces à l'aide de petites pieces assemblées, dont on s'emerveillait a la fin du xviIle siecle. Le plus célebre exemple en est la première coupole de la halle aux blés de Paris.

44. D1 15 (3). 
discipline du travail, et l'on peut remarquer dans toutes les relations dont nous disposons, que c'est à l'évidence ce qui frappa les imaginations.

\section{LE TEMPS : LA DISCIPLINE DU TRAVAIL}

Pour le Ministère, la meilleure façon d'obtenir la diligence souhaitté était de multiplier les épreuves : des ouvriers on n'attend pas qu'ils comprennent, mais qu'ils s'accoutument, ce que seule permet la répétition.

« Lorsque toutes les pièces du vaisseau seront entièrement achevées, écrivait Colbert à de Seuil, il faudra que vous le fassiez monter deux, trois ou quatre fois en vostre presence et que vous ne vous estonniez pas si dans les premières fois les charpentiers sont longtemps, parce que dans l'usage ils s'apprendront a le monter dans le temps necessaire et mesme plus promptement $\gg 45$.

Formule étonnante qu'éclaire cette autre, énoncte quelques mois plus tard : il faut par ces épreuves « accoustumer les ouvriers qui doivent travailler à l'ordre et à la promptitude avec laquelle chacun doibt travailler $"{ }^{40}$.

Le caractère inédit de ces montages ne permet pas d'évaluer à l'avance le temps nécessaire. Il va dépendre pour une bonne part de l'adaptation de chaque ouvrier à une situation où la succession des operations se trouve nécessairement modifiée. C'est de leur coordination que naîtra la rapidité recherchée, ce qui justifie la proposition d'Arnoul : plutôt que de se fier à la seule accoutumance par répetition, il demanda l'autorisation de faire d'abord construire un vaisseau de même proportion à l'ordinaire, afin de « ranger mes ouvriers, leur apprendre leur poste et faire mieux comprendre aux Mes de quelle manière je m'imagine que l'on s'y peut prendre pour approcher la diligence que vous souhaitez $\$ 47$.

De cet ordre, l'Intendant est l'inventeur. Conçu, essayé, puis transcrit dans le Mémoire qui rendait compte de la première épreuve, il faisait l'objet des critiques du Ministère qui suggérait des modifications, demandant alors à chaque Intendant de s'appliquer « à observer l'ordre qui luy a esté prescrit $"{ }^{48}$. Des divergences apparurent entre les Ports et la Cour sur deux points : le travail de nuit et la rotation des équipes.

45. B2 39 (449).

46. Le 26 déc. 1678 , in $\mathrm{B} 239$ (637).

47. Lettre du 17 janv. 1679, in B3 32 (40).

48. Lettre à Denuin, mars 1679, in B2 40 (245). 
L'épreuve commencera à trois heures du matin. La nuit, le travail sera poursuivi, le chantier éclairé par des chandelles et des flambeaux ${ }^{49}$. Denuin passa outre cette dernière injonction de Colbert, de même que de Seuil qui s'expliqua :

" Comme il est de necessité, pour la facilité des ouvriers, à reconnoistre les membres et autres pièces de bois et leurs places et de les marquer par chiffre, il sera difficile de bien advancer en pareil travail pendant les heures de la nuit, quelque jour que les flambeaux puissent donner à cause desdites marques qui feroient passer beaucoup de temps avant d'estre conneuës ce qui seroit aussy si on en prenoit une pour l'autre que cela pourroit prolonger le temps de construction $»$ so.

Quant au repos des hommes, il était assuré par le renouvellement des escouades qui composaient les divisions, sauf à Brest où les 204 ouvriers travaillaient ensemble de telle sorte que, pendant deux heures, le chantier était arrêté. Pour accélérer le montage, Colbert proposa à de Seuil d'augmenter le nombre des ouvriers de cinquante hommes, qui auraient pris le travail pendant le repos des divisions. $\grave{A}$ quoi de Seuil rétorqua qu'« il serait certes d'un grand secours, mais il faudroit pour les conduire quelqu'un du Me ou des Contremaistres qui auront besoin de prendre haleine quand ils auront travaillé sept ou huit heures, sans relâche, estant necessaire qu'ils soient toûjours ensemble " ${ }^{\text {sI }}$. La rigidité de cette organisation du travail ne peut manquer d'étonner : Rochefort, et surtout Toulon, offrant dans le même temps des solutions plus astucieuses, on comprend mal que Colbert ait toléré ce système, sauf à penser qu'il s'agissait de faire l'épreuve d'une autre manière.

A Toulon, les effectifs furent beaucoup plus importants : 512 charpentiers et 78 perceurs dans l'épreuve de juillet, 768 ouvriers, le 6 novembre ; sans doute, cet accroissement du nombre des hommes s'explique-t-il parce que dans cette dernière épreuve le vaisseau fut calfaté, mâté, agréé et mis à l'eau. Dans les deux cas, pour la construction,

" tous les ouvriers et tout l'ouvrage avait été partagé entre les quatre principaux Mes Bastisseurs de la Marine qui faisoient quatre divisions differentes et chacun d'eux avoit soubs luy deux soub maistres l'un pour l'avant et l'autre pour l'arrière, chaque division estoit partagé en huit escouädes commandées chacune par un chef et composée de seize charpentiers et de

49. Lettre à Denuin du 18 avr. 1679, in B2 40 (303).

50. Relation de l'épreuve du 4 août 1679 , in Di 15 (3).

51. Ibid. Chaque division était sous les ordres d'un maître-charpentier. 
quatre perceurs. [Cependant] de ces huit escouädes il y avoit toûjours deux qui devoient se reposer et qui se devoient relever successivement de deux heures en deux heures $\$ 32$.

Quant au « département » des ouvriers, nous savons qu'à Brest ceux-ci

« furent partagés également pour chacun des deux costés dudit vaisseau, et ceux de chaque costé partagés en trois escouädes deux desquelles estoient employées l'une à l'avant et l'autre avoit à mettre en place tous les membres dudit costé, et la troisième à porter tous les bois suivant son rang et sa marque $\gg$ s3.

Les épreuves successives n'avaient pas pour seul objet d'accoutumer les ouvriers. Elles permettaient de mieux coordonner les travaux dans le temps. On devait donc procéder à de véritables minutages des différentes opérations,

« la principale application de l'Intendant estant de monter en deux jours de temps au plus. Pour cet effet, se doibt observer un grand ordre et marquer les heures et les momens du travail pour parvenir tousjours a diminuer le tems qui sera employé à cette construction $\$ 54$.

Ce minutieux ordonnancement fit l'admiration de chacun à Toulon, si l'on en croit la relation qui nous est parvenue :

« Tous les ouvriers [...] estoient à l'arsenal avant le jour, ils y entendirent la messe [...] en suitte de quoy chacun se fut ranger de luy mesme au poste qui luy avoit esté marqué, le jour auparavant pour attendre que le signal leur fust donné ; ils furent ainsy sans rien faire jusqu'à quatre heures et pour lors au son des trompettes et des tambours on vit pres de cinq cents hommes remuer dans un moment, et tous à la fois comme dans un concert faisant chacun une maneuvre differante avec un ordre et un silence que l'on ne peut pas s'imaginer à moins que d'y avoir esté present ; c'estoit aussy ce qui leur avoit esté recommandé tres expressément et sur quoy l'on avoit pris toutes les precautions imaginables, d'autant qu'il s'agissoit de bien placer d'abord les membres de ce vaisseau ce qui ne pouvoit se faire que par un accord general de tous les ouvriers ensemble ; ensuitte de quoy ils se separèrent en quatre et chacun soubs son chef se mit à travailler à l'envy l'un de l'autre au poste qui luy avoit esté destiné, de sorte que ce vaisseau qui n'avoit esté commencé qu'à quatre heures se trouva entièrement finy devant les onze heures du matin. ")

52. Relation du 14 juil. 1679 , in D1 15 (4).

53. D1 15 (3).

54. B2 40 (303). 
Dans ce concert des gestes et des mancuvres,

" tous les coups qui se donnoient n'en paroissoient qu'un et le travail se faisoit d'une si grande vitesse qu'on croyoit l'ouvrage finy plustost que la main qui l'avoit fait [...]. Ce zele extraordinaire et cette diligence sans pareille estoient cependant conduitte d'une manière que le travail s'acheva sans qu'il se rompît aucune pièce et sans qu'aucun des ouvriers se fit le moindre mal quoiqu'il y en eust pres de sept cents qui estoient employés dans ce travail tant on avoit pris de precautions sur ce subject. "

Cette belle discipline des hommes consista à Toulon, non pas tant à les presser au travail, qu'à modérer leur ardeur. Elle était si grande, nous dit-on, que ceux qui devaient assurer la relève,

" croyant que le vaisseau s'eslevoit à leurs yeux pendant qu'ils avoient les bras croises, après avoir fait plusieurs instances pour qu'on leur permît de travailler, s'en [vinrent] d'eux-mesmes à leur departement, pour avoir du moins quelque part à la gloire de leurs camarades; de sorte que l'on fust contraint de prier les plus fatigués de deposer, ce qu'on ne put jamais obtenir que sur un petit nombre, les trois quarts s'estant fait un point d'honneur de ne point boire ny manger, que tout le vaisseau ne fust finy, tant ils estoient transportés du desir d'avoir bientost fait leur ouvrage." "

Le « transport » faillit tourner à l'émeute

" et s'estant trouvé un seul qui laissa tomber une masse, faute de force, les autres croiant qu'il n'alloit pas de bon pied et l'accusant de vouloir trahir la Nation se jetèrent aussy tost sur luy pour l'assommer et l'auroient jeté du haut en bas d'un eschafaut sans un Commissaire qui les en empescha. "

\section{Cette ardeur croissait avec le grade :}

« Les Maistres et chefs d'escouâde par dessus tous les autres estoient si fort animés que la violence du travail, jointe a la chaleur de la saison dans ce climat, leur ayant fait monter le sang au visage le leur faisoit paroistre tout violet, leurs levres estoient ecumantes et noires, et plusieurs en auroient perdu la parolle, en sorte qu'il y en a un entre autres appelé Joseph Gues qui ne peut pas dire encore un seul mot, bien qu'il y ait desja deux jours de passés depuis cette action $\$ 55$.

C'était bien une action, un combat. Toutes les marques et clichés y sont, depuis l'honneur de défendre la nation dont on est, jusqu'à la folle

55. D1 15 (4). 
ardeur de ces " soldats français " que l'on discipline à grand mal. Certes, l'écrivain grossit le trait pour la plus grande gloire de leur chef : Arnoul. Cependant, comment celui-ci obtint-il une telle tension de ses hommes ${ }^{36}$ ?

On est loin de la belle parade, tout aussi militaire, que nous annonce l'écrivain à la suite de ces images de violence, jugeant que toutes ces circonstances donneraient sans doute au public quelque impatience de savoir quel était l'ordre qui s'était observé.

" Il eust esté bien difficile d'empescher la confusion dans un si grand nombre de gens veu qu'il devoit y avoir toûjours pres de cinq cent hommes dans le travail sans compter les chefs, si on ne les avoit habillés differamment. Le Sr Coulomb, Maistre Bastisseur du Navire qui est celuy qui a fait et conduit le vaisseau dont il est question, et qui devoit faire en cette occasion le costé droit, estoit habillé de bleu avec toute sa division, celle du Sr Chapelle, autre bastisseur de navire qui devoit faire le costé gauche estoit toute blanche, celle de Me Coulomb le fils qui devoit faire le fond de cale avoit le caleçon bleu et la chemise blanche, et celle de Me Audibert qui devoit faire les ponts avoit le caleçon blanc et la chemise bleue ; les escouädes de chaque division se distinguoient de plus par le moien d'un ruban de couleur differante, les chefs avoient mesme une marque particulière et les perceurs estoient distingués des charpentiers en ce qu'ils avoient chacun une masse à la main avec une gibessière à leur costé pour tenir les cloux et leurs outils. »

Pour mener l'action et s'assurer le contrôle du chantier et de l'atelier, Arnoul avait sous lui quatre commissaires de marine et neuf écrivains qui avaient rapport aux quatre Me charpentiers pour huit d'entre eux, le neuvième, au Me d'équipage qui devait passer les palans. L'Intendant se servait d'eux

« pour sçavoir à tout moment l'estat du travail dans les endroits où il n'estoit pas, pour y donner les ordres et fournir au besoin, supposé qu'il eust manqué quelque chose qu'il n'eust pas preveu, mais ce qu'il y a de plus admirable et de plus surprenant c'est que l'on n'a manqué de rien et toutes les choses qui estoient necessaires pour le travail avoient esté si bien dispersées sur l'atellier, que tout se trouva par les ordres de Monsieur Arnoul dans l'endroit de sa destination, sans qu'on fust obligé de rien aller chercher ailleurs ".

56. Des promesses de récompense devaient y avoir leur part. Dans une lettre du 4 juin 1679 à Denuin, donc après la première épreuve, et pour tenter d'accélérer le travail, Colbert propose : « A l'esgard des deux Mes charpentiers qui ont basty cette fregate s'ils veulent entreprendre de la bastir devant Sa Majesté en vingt heures de temps, elle sera donnée une chaisne d'or de six vingt pistoles au père et une autre de soixante au fils ", in $\mathrm{B} 241$ (409). 
Compte non tenu de la belle discipline militaire que l'on nous décrit, on peut penser qu'Arnoul ne put faire manœuvrer ces hommes tous ensemble, et en particulier ne put faire travailler en même temps au fond du vaisseau et sur les côtés, que parce qu'il disposait de la halle : on pouvait faire venir les pièces nécessaires jusqu'au-dessus du vaisseau en construction ${ }^{57}$. L'extraordinaire réussite des épreuves de Toulon - huit fois moins de temps qu'à Rochefort - justifie son obstination à l'obtenir.

En portant tout particulièrement ses efforts sur la maîtrise de l'espace pour gagner du temps, Arnoul avait pris le bon parti. Le fait que Du Maitz ait envoyé dans tous les Ports une relation sur l'arrangement des apparaux et des pièces dans la halle, souligne le caractère exemplaire que Toulon voulait imprimer à ces aménagements. Le concours ardent que l'on obtint des ouvriers, la violence de leurs efforts pour réussir cet exploit témoignent d'une volonté de parvenir coûte que coûte à emporter l'admiration : Arnoul était l'inventeur d'une organisation du travail sans commune mesure avec ce qui s'était fait jusqu'alors. Tout conspira ainsi à la glorification de cet Intendant, à laquelle se consacre la relation du 14 juillet. Véritable apologie, elle ne s'adresse pas of ficiellement à la Cour, mais est un appel à l'opinion publique en sa faveur ${ }^{58}$. Il faut dire qu'il en avait grand besoin ${ }^{59}$. Cependant, on peut se demander si cette conspiration réussie du personnel de la Marine à Toulon, à l'occasion de ces montages, n'a pas hâté la disgrâce de l'Intendant qui menaçait.

On sait les difficultés que Colbert eut avec ses Intendants ${ }^{60}$. Sans doute faut-il y voir les effets de la situation qui leur était faite entre l'organisation de la Marine et les pouvoirs locaux. Sans compter les conflits

57. C'est en 1786, selon Vincent Brun, que l'idée fut reprise d'établir des cales couvertes pour mettre les vaisseaux en construction. Malouet, Intendant de Toulon, fit procéder aux études préalables et envoya un devis à la Cour. Un toit d'environ 60 pieds de haut était soutenu par des poteaux. Malouet cependant signala le danger que pouvait faire courir un coup de vent. On construisit des cales à Rochefort, qui semblent avoir résisté aux intempéries, puis à Brest (1686) et Lorient. Cf. V. BRUN, Guerres maritimes de la France, port de Toulon, Paris, 1861, t. II, p. 105.

58. Il y est fait brievement allusion dans la Gazette de France de Toulon, le 18 juil. 1679.

59. Sans entrer dans le détail de son histoire, il faut savoir qu'Arnoul et Seignelay avaient été élevés dans le mème college, et que Colbert avait chargé Arnoul père, Intendant de Marseille, d'initier Seignelay aux problèmes de la Marine. Quelles qu'aient été les raisons de leurs différends, durant la période qui nous intéresse Seignelay poursuivit Arnoul avec une violence qui étonna tout le monde. En particulier, il l'accusa d'être responsable de la destruction de l'escadre de Tourville par la tempéte. Les radoubs avaient été faits à Toulon, et seule " l'horrible négligence " de l'Intendant en cette occasion pouvait, selon Seignelay, expliquer le désastre. Malgré Duquesne et Tourville lui-même qui soutinrent Arnoul jusqu'au bout, la disgráce fut prononcée le 26 novembre 1679. Envoyé au Havre, il fit par la suite, une carrière fort honorable.

60. Ainsi, Denuin fut démis de ses fonctions pour malversation. 
toujours latents, sinon déclarés, avec le corps des Fortifications ${ }^{61}$. Que ces difficultés, somme toute inévitables, soient alors exacerbées, ne doit pas étonner : dans ces années 1670-1680, on est à l'heure des innovations qui engagent la Marine dans un système de fonctionnement qui va marquer son histoire à venir.

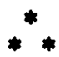

Si l'on revient aux quelques remarques générales hasardées au début de ce texte, de quoi Colbert voulut-il faire l'expérience en se plaçant dans des conditions aussi exceptionnelles ? En quoi ces dernières pouvaientelles devenir instructives ? Qui voulait-on instruire ${ }^{62}$ ?

Pour répondre à la première question, on peut se référer à l'histoire des techniques constructives : situer ces montages par rapport au chef-d'œuvre artisanal, moyen contemporain de faire la preuve de sa maîtrise, et à la production en série : ce que suggère l'idée de ces " préfabrications".

Les frégates réalisées n'avaient rien d'exceptionnel. Conçues par les charpentiers du port, les méthodes, toutes traditionnelles, d'élaboration de leurs formes ne sont pas modifiées par ces montages : elles les précèdent. Toujours du point de vue de l'objet réalisé, la production n'est pas davantage de série. Chaque vaisseau est différent selon le port. On propose, d'ailleurs, de fabriquer une frégate de 26 à 30 pièces de canon. Le choix devait se faire sur place, en fonction des bois dont on disposait.

À l'évidence, le défi lancé par Colbert est démarqué de celui de Sardam. Colbert s'y réfère souvent, nous l'avons vu ${ }^{63}$. Dans son Dictionnaire de la Marine, paru en 1702, Aubin y apporte des précisions :

61. Il semble que Vauban eut d'excellents rapports avec Arnoul durant cette période. Comme nous l'avons vu, il appuya son projet de forme flottante en bois. Toujours d'apres l'écrivain de Toulon, c'est Arnoul qui proposa le plan du nouvel arsenal auquel Vauban applaudit, " bien loin d'y rien changer " et qu'il transmit à la Cour. Dans son deuxième Mémoire sur Toulon, Vauban dit encore de ce plan que « tout son dessein est le père de tous les autres " (21 mars 1681).

62. Qu'elle ait la vertu d'une expérience, c'est ce que conforte la remarque qu'il fit à Arnoul père en lui demandant de procéder à des montages rapides de galères préfabriquetes : « Je serais bien ayse [...] que cette expérience se fist en la présence de mon fils, afin qu'elle pust servir à son instruction ", in P. ClémeNT, op. cit. supra n. 1, t. III, p. 299.

63. Cf. op. cit. supra n. 12. Cf. également, "Lettre au Sr Leger controleur général de l'arsenal de Toulon ", mars 1670, in P. CLÉMENT, op. cit. supra n. 1, t. III, p. 216, n. 1 : "Nous voyons les Hollandois tenir, dans le seul village de Sardam, les bois nécessaires pour bastir quatre ou cinq cents vaisseaux, par le bon ordre qu'ils y apportent." 
« On tient communément en Hollande que le Bourg de Sardam où est la grande fabrique des vaisseaux, peut, pendant une campagne entière, fournir tous les jours un vaisseau, en donnant tour à tour un du plus haut rang jusques au cinquième et plus bas rang, pourvu qu'on soit averti, \& qu'on en soit convenu quatre ou cinq mois auparavant $" 64$.

L'information est intéressante : il ne s'agit pas davantage de produire une série de vaisseaux identiques. Il faut ajouter immédiatement que, pour les charpentiers, ce serait une moindre perfection. En quoi ils se conforment à l'avis des officiers de marine : pour son utilité, une flotte doit être diversifiée. On ira jusqu'à dire que, dans la guerre, il faut disposer de vaisseaux dont les comportements soient, en quelque sorte, individualisés ; ainsi, dans n'importe quelle situation on dispose d'un bâtiment qui répond exactement à la mission envisagée. La perfection visée est contraire à l'idée même de série. On voit ici avec quelle extrême prudence il convient de manier nos catégories. Même lorsque l'on en vient, à l'époque de nos montages, à essayer d'appréhender le vaisseau comme un ensemble de fonctions dont il faut aménager les combinaisons, l'archétype qui prévaut encore souvent et qui modèle l'idée de fonction est celui d'une nature par essence diverse, c'est-à-dire dont la perfection est l'adéquation à la singularité des cas. Entre l'exception et la règle, les jeux sont multiples, et cela vaut pour la mise en scène de nos montages de vaisseaux.

À cet égard, le modèle de Sardam est intéressant : les habitants de ce bourg firent leur proposition à l'occasion d'une guerre ${ }^{65}$. A situation d'exception, réponse exceptionnelle. L'exploit demandé à Brest, Rochefort et Toulon, serait une simulation : concevoir et expérimenter une production adéquate aux circonstances d'une guerre ${ }^{66}$. Alors, l'expérience peut être instructive.

Instructive, d'abord, de l'ordre à tenir si l'on veut devenir « compétitif » : lorsque Colbert évoque Sardam, c'est comme exemple de ce que l'on peut obtenir si l'on « examine avec soin tout ce qui peut contribuer à construire, équiper et armer les vaisseaux avec facilité et en peu de temps $"{ }^{67}$. La même conception de l'ordre doit présider aux maga-

64. Article « Construction 》.

65. Cf. op. cit. supra n. 12, p. 21.

66. C'est aussi une manière de résoudre un problème crucial : celui de la conservation des vaisseaux en temps de paix.

67. Cf. P. Clement, op. cit. supra n. 1, t. III, p. 295 : « Mémoire sur le règlement à faire pour la police générale des arsenaux de Marine ", oct. 1670. En général, il faut faire " en sorte que les estrangers soyent portés à venir voir "; cf. ibid., "Instructions pour le Sr Matharel... ", p. 233. 
sinages et à l'organisation du travail. La puissance de cet ordre tient à sa cohérence fonctionnelle. Celle-ci est à la fois grandiose et dérisoire, et ce jusqu'aux clous : " il n'y a rien, écrit Colbert, qui puisse tant marquer la grandeur et la puissance de Sa Majesté à l'égard de la Marine que la grande quantité de bois, masts, canons, armes, munitions [...] et de tenir le tout bien rangé $"{ }^{68}$. Sur ces rangements, il détaille, revenant sans trêve. Or, si rien ne marque tant la grandeur de S.M., le moindre désordre en est la dérision. Quand on sait la gabegie qui régnait dans les ports, on peut se demander si la mise en scène des montages de vaisseaux n'avait pas pour objet de faire l'expérience d'une grande discipline. En quelque sorte, prouver la marche en marchant et persuader ainsi les acteurs. La solennité qui entoura les épreuves, est un artifice qui a le pouvoir bien connu de rectifier la conduite des hommes et de faire naître la confiance ${ }^{69}$. Mais on peut dire aussi bien qu'en ne faisant varier qu'une seule des conditions de la construction, le temps, - de préparation et d'exécution -, Colbert exigeait que l'on reconsidère et expérimente de nouvelles combinaisons de toutes les autres conditions, introduisant ainsi le doute dans des esprits dont on ne cessera de brocarder l'intolérance à toute espèce de nouveauté ${ }^{70}$.

Ces deux interprétations ne sont pas contradictoires. Introduire le doute sur la nécessité de ce qui existe, et développer la confiance en une démarche prospective est tout un pour qui vise à concevoir et réaliser de nouveaux possibles. Dans la Marine, en 1679 , on ne pouvait guère aller plus avant.

Hélène VÉRIN, C.N.R.S.

\section{Ibid.}

69. Il en use auprès des entrepreneurs. Dans ses «Instructions pour le Chevalier de Clerville, commissaire aux fortifications ", 11 octobre 1670, in P. CLÉMENT, op. cit. supra n. 1, t. V, p. 33, Colbert recommande: « pour que rien ne manque aux formes qui peuvent esclaircir la rectitude qui doit être gardée en ces ouvrages "), que l'on procède aux adjudications " dans des formes si solennelles que personne ne puisse soupçonner aucune fraude ".

70. Aubin prétendra des officiers de Marine que, plus ils sont intelligents, moins ils l'acceptent. Pierre Bouguer, dans son Traité du navire, de sa construction et de ses mouvements, Paris, 1746, accusera tour à tour les bornes de l'esprit, un intérêt mal compris, une déficience dissimulée des savoirs. Quant à Henri-Louis DUHAMEL DU MONCEAU, c'est en cartésien systématique qu'il s'appliquera, dans le Traité pratique de la construction des vaisseaux, Paris, 1758, à développer " un doute méthodique » pour combattre les préjugés qui règnent dans la construction navale. 